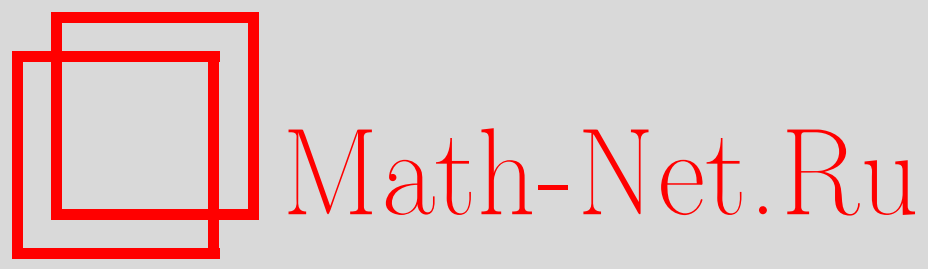

С. М. Гусейн-Заде, Ф. Дельгадо, А. Кампильо, О монодромии особенности плоской кривой и ряде Пуанкаре ее кольца функций, Функи. анализ и его прил., 1999, том 33, выпуск 1, 66-68

DOI: https://doi.org/10.4213/faa340

Использование Общероссийского математического портала MathNet.Ru подразумевает, что вы прочитали и согласны с пользовательским соглашением

http://www.mathnet.ru/rus/agreement

Параметры загрузки:

IP : 54.237 .59 .107

26 апреля 2023 г., 02:32:30

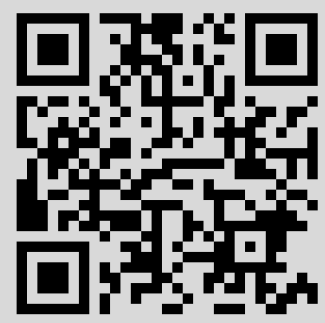




\section{О монодромии особенности плоской кривой и ряде Пуанкаре ее кольца функций ${ }^{\star}$}

(C) 1999. С. М. ГУСЕЙН-ЗАДЕ, Ф. ДЕЛЬГАДО, А. КАМПИЛЬО

Целью настоящей заметки является следующее утверждение: дзета-функция преобразования классической монодромии неприводимой особенности плоской кривой совпадает с рядом Пуанкаре кольца функций на ней.

Пусть $(C, 0) \subset\left(\mathbb{C}^{2}, 0\right)$ - неприводимая особенность плоской кривой, задаваемой уравнением $f=0, f:\left(\mathbb{C}^{2}, 0\right) \rightarrow(\mathbb{C}, 0)$ - росток голоморфной функции с изолированной критической точкой в начале координат. Пусть $h_{f}: V_{f} \rightarrow V_{f}$ - преобразование классической монодромии особенности $f$, действующее на ее слое Милнора $V_{f}$ (см. [2]). Дзета-функция преобразования $h: X \rightarrow X$ топологического пространства $X$ (для корректности имеющего конечномерные гомологии) - это рациональная функция

$$
\zeta_{h}(t)=\prod_{q \geqslant 0}\left[\operatorname{det}\left(\mathrm{id}-\left.t \cdot h_{*}\right|_{H_{q}(X ; \mathbb{R})}\right)\right]^{(-1)^{q+1}} .
$$

Пусть $\zeta_{f}(t)$ - дзета-функция преобразования классической монодромии $h_{f}: V_{f} \rightarrow V_{f}$ особенности $f$.

ЗАмечАниЕ. Это - определение дзета-функции из [1]. Дзета-функция, определенная в [2], является обратной к ней.

Пусть $\mathscr{O}_{\mathbb{C}^{2}, 0}$ - кольцо ростков голоморфных функций в начале координат на комплексной плоскости $\mathbb{C}^{2}$, и пусть $\mathscr{O}_{C}=\mathscr{O}_{\mathbb{C}^{2}, 0} / f \mathscr{O}_{\mathbb{C}^{2}, 0}$ - кольцо ростков функций на кривой $C$. Пусть $p:(\mathbb{C}, 0) \rightarrow\left(\mathbb{C}^{2}, 0\right)$ - униформизация (параметризация) кривой $C$, т.е. росток аналитического отображения, образ которого совпадает с $C$ и которое является изоморфизмом между $\mathbb{C}$ и $C$ вне начала координат. Для ростка $\varphi \in \mathscr{O}_{C}$ обозначим через $v(\varphi)$ порядок функции $\varphi \circ p:(\mathbb{C}, 0) \rightarrow \mathbb{C}$ в нуле, т. е. степень главного члена в ее степенном разложении $\varphi \circ p(\tau)=a \cdot \tau^{v(g)}+$ члены более высокой степени, $a \neq 0$; $v(0)$ полагается равным бесконечности. Функция $v(\cdot)$ определяет фильтрацию кольца $\mathscr{O}_{C}: F_{i}=\left\{\varphi \in \mathscr{O}_{C}: v(\varphi) \geqslant i\right\}$. Пусть $L(t)-$ ряд Пуанкаре этой фильтрации: $L(t)=\sum_{i=0}^{\infty} c_{i} t^{i}$, где $c_{i}=\operatorname{dim}_{\mathbb{C}}\left(F_{i} / F_{i+1}\right)$. Легко показать (см. ниже) что ряд $L(t)$ сходится в окрестности начала координат и определяет рациональную функцию переменной $t$ (также обозначаемую здесь через $L(t))$.

TeOpema. $\zeta_{f}(t)=L(t)$.

ДокаЗАТЕЛьСтво. Пусть $\pi:(X, D) \rightarrow\left(\mathbb{C}^{2}, 0\right)$ - минимальное разрешение кривой $C$ с помощью последовательности $\sigma$-процессов в особых точках

* Работа первого автора выполнена при частичной поддержке грантов Iberdrola, РФФИ-98-01-00612 и INTAS-97-1644; работа последних двух авторов (F. Delgado, A. Campillo) выполнена при частичной поддержке гранта DGICYT PB94-1111-C02-01 и гранта Кастильи и Леона VA51/97. 
([2]; под этим понимается разрешение кривой как вложенной в комплексную плоскость, которому в англоязычной литературе соответствует термин «embedded resolution».) Двойственный граф этого разрешения показан на рис. 1. Вершины $\alpha$ этого графа соответствуют неприводимым компонентам $E_{\alpha}$ исключительного дивизора $D$ разрешения (эти компоненты изоморфны комплексным проективным прямым). Две вершины графа соединены ребром, если соответствующие компоненты пересекаются. Стрелка соответствует собственному прообразу кривой $C$. Здесь $g$ - количество пар Пюизо $\left(m_{j}, n_{j}\right)$ кривой $C[2]$. Пусть $v^{\alpha}$ - кратность поднятия $f \circ \pi$ функции $f$ (уравнения кривой $C$ ) на пространство $X$ разрешения вдоль компоненты $E_{\alpha}$ исключительного дивизора, соответствуюшей вершине $\alpha$ двойственного графа разрешения. Пусть $\bar{\beta}_{j}=v^{a_{j}}, \gamma_{j}=v^{b_{j}}$ для вершин $a_{j}$ и $b_{j}$, указанных на рис. 1.

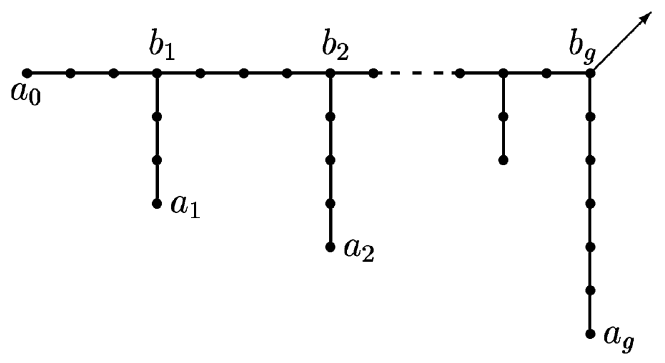

Рис. 1. Двойственный граф разрешения кривой $C$

В соответствии с формулой Н. А'Кампо [1] дзета-функция $\zeta_{f}(t)$ особенности $f$ равна $\prod_{\alpha}\left(1-t^{v_{\alpha}}\right)^{-\chi\left(E_{\alpha}^{0}\right)}$, где $E_{\alpha}^{0}$ - компонента $E_{\alpha}$ без точек пересечения с другими компонентами полного прообраза кривой $C$ (т.е. с компонентами исключительного дивизора $D$ и с собственным прообразом кривой $C$ ), а $\chi(\cdot)$ - эйлерова характеристика. Эйлерова характеристика $\chi\left(E_{\alpha}^{0}\right)$ равна нулю, если вершина $\alpha$ не совпадает ни с одной из вершин $a_{j}, b_{j}, \chi\left(E_{a_{j}}^{0}\right)=1$, $\chi\left(E_{b_{j}}^{0}\right)=-1$. Таким образом, получаем

$$
\zeta_{f}(t)=\frac{\prod_{j=1}^{g}\left(1-t^{\gamma_{j}}\right)}{\prod_{j=0}^{g}\left(1-t^{\bar{\beta}_{j}}\right)} .
$$

Полугруппа $S$ кривой $C$ - это множество целых чисел $i \in \mathbb{Z}_{\geqslant 0}$, для которых существует росток $\varphi \in \mathscr{O}_{C}$ с $v(\varphi)=i$. Известно (см. [3, 4]), что:

(1) $c_{i}=\operatorname{dim}_{\mathbb{C}}\left(F_{i} / F_{i+1}\right)$ отлично от нуля тогда и только тогда, когда $i-$ элемент полугруппы $S$ кривой $C$; в этом случае $c_{i}=1$;

(2) множество $\left\{\bar{\beta}_{j}: j=0,1, \ldots, g\right\}$ является минимальным семейством образующих полугруппы $S$;

(3) целое число $\gamma_{j}$ кратно $\bar{\beta}_{j}(j=1, \ldots, g)$, т.е. $\gamma_{j}=m_{j} \bar{\beta}_{j}$ (в действительности $m_{j}$ - члены пар Пюизо кривой $C$ );

(4) любой элемент $i$ полугруппы $S$ единственным образом представим в виде $i=k_{0} \bar{\beta}_{0}+\sum_{j=1}^{g} k_{j} \bar{\beta}_{j}$ с $k_{0} \geqslant 0,0 \leqslant k_{j}<m_{j}$ при $1 \leqslant j \leqslant g$. 
Поэтому имеем

$$
\begin{aligned}
L(t) & =\sum_{i=0}^{\infty} c_{i} t^{i}=\sum_{i \in S} t^{i}=\left(\sum_{k_{0}=0}^{\infty} t^{k_{0} \bar{\beta}_{0}}\right) \cdot \prod_{j=1}^{g}\left(\sum_{k_{j}=0}^{m_{j}-1} t^{k_{j} \bar{\beta}_{j}}\right) \\
& =\frac{1}{1-t^{\bar{\beta}_{0}}} \cdot \prod_{j=1}^{g} \frac{1-t^{m_{j} \bar{\beta}_{j}}}{1-t^{\bar{\beta}_{j}}}=\frac{\prod_{j=1}^{g}\left(1-t^{\gamma_{j}}\right)}{\prod_{j=0}^{g}\left(1-t^{\bar{\beta}_{j}}\right)}=\zeta_{f}(t),
\end{aligned}
$$

что и требовалось доказать.

Примечание при корректуре. Авторами получен аналог утверждения теоремы для плоской алгебраической кривой с одной ветвью на бесконечности.

\section{ЛитеРАТУРА}

1. A'Campo N. Comment. Math. Helv., 50, 233-248 (1975). 2. Арнольд В. И., Варченко А. Н., Гусейн-Заде С. М. Особенности дифференцируемых отображений. T. II. Наука, M., 1984. 3. Campillo A. Algebroid curves in positive characteristic. Lecture Notes in Math., vol. 813, Springer-Verlag, Berlin-Heidelberg-New York, 1980. 4. Teissier B. В кн.: Zariski $O$. Le problème des modules pour les branches planes. Hermann, Paris, 1986, Appendice.

Московский государственный университет e-mail: sabir@mccme.ru

Поступило в редакцию University of Valladolid (Spain)

e-mail: campillo@cpd.uva.es, fdelgado@cpd.uva.es

УдК 517.55

\section{О размерности группы, транзитивно действующей на гиперповерхности в $\mathbb{C}^{3}$ *}

(C) 1999. А. В. ЛободА

В работе [1] Э. Картан описал все вещественные гиперповерхности двумерных комплексных пространств, на которых транзитивно действуют группы Ли голоморфных преобразований. Ниже обсуждаются размерности аналогичных групп Ли в пространствах трех комплексных измерений.

Для однородной гиперповерхности $M$, обладающей такой группой $\operatorname{Aut}(M)$, размерность $\operatorname{dim} \operatorname{Aut}(M)$ складывается из $\operatorname{dim} M=5$ и размерности группы $\operatorname{Aut}_{\xi}(M)$ голоморфных автоморфизмов гиперповерхности $M$ с неподвижной точкой $\xi \in M$. Все рассмотрения ниже связаны с последней группой. При этом используется техника многомерного комплексного анализа и, в первую очередь, аппарат локальных нормальных форм. В связи с этим будем считать, что все поверхности вложены в пространство $\mathbb{C}^{3}$, а элементы

* Настоящая работа выполнена при поддержке Российского фонда фундаментальных исследований (проект № 96-01-01002). 